\title{
Photothermal therapy in a murine colon cancer model using near-infrared absorbing gold nanorods
}

\author{
Glenn P. Goodrich \\ LiLi Bao \\ Kelly Gill-Sharp \\ Krystina L. Sang \\ James Wang \\ J. Donald Payne \\ Nanospectra Biosciences, Incorporated \\ 8285 El Rio Street, Suite 150 \\ Houston, Texas 77054
}

\begin{abstract}
The photothermal ablation of solid tumors using exogenous, near-infrared (NIR)-absorbing nanoparticles has been previously investigated using various preclinical models and is currently being evaluated in the clinic. Here, we evaluate the circulation kinetics, preliminary toxicity, and efficacy of photothermal ablation of solid tumors using gold nanorods systemically delivered and passively accumulated in a murine subcutaneous colon cancer model. Tumored animals were infused with nanorods followed by the percutaneous illumination of the tumor with an 808-nm laser. Control groups consisted of laser-only, nanorod-only, and untreated tumored animals. The survival of the treated and control groups were monitored for 60 days post-treatment. The survival of the photothermally treated group was statistically longer than the control groups, with approximately $44 \%$ tumor free through the evaluation period. Histopathology of the major organs of animals infused with nanorods did not indicate any significant toxicity at 60 days post-treatment. Particle biodistribution was evaluated by elemental analysis of the major organs of untumored mice at 1, 7, and 30 days after infusion with nanorods. Elemental analysis indicates nanorod clearance from the blood and retention by the reticuloendothelial system. This study indicates that gold nanorods are promising agents for photothermal ablation of solid tumors. () 2010 Society of Photo-Optical Instrumentation Engineers. [DOI: $10.1117 / 1.3290817]$
\end{abstract}

Keywords: nanorod; near-infrared; photothermal therapy; ablation; nanoparticle; cancer.

Paper 09277RR received Jul. 8, 2009; revised manuscript received Nov. 10, 2009; accepted for publication Nov. 11, 2009; published online Jan. 15, 2010.

\section{Introduction}

The ablation of solid tumors has been investigated using a variety of energy methods, including endogenously absorbed laser energy ${ }^{1-3}$ and radio-frequency energy. ${ }^{4,5}$ The tumor specificity of these approaches is generally limited by the precision of pretreatment imaging, the precision of applicator placement, and the energy-deposition shape of the applicator used (generally spherical or conical). As a result, the treatment of tumors surrounding or adjacent to critical structures is problematic, the possibility of residual positive margins may limit use, and the ablation of surrounding healthy tissue may result in cosmetically or medically unacceptable outcomes.

Recently, the use of submicron- or nano-sized particles has been investigated to provide a higher level of precision for ablative applications. The objective of particle-based therapy is to select or design the particle (by size, shape, or other properties) to specifically accumulate in the tumor and not in normal tissue. The particles can be delivered to the tumor either passively, accumulating in the tumor through vascular fenestrations (the enhanced permeability and retention effect, EPR $),{ }^{6-8}$ or actively targeted to receptors on tumor or tumor-

Address all correspondence to: Glenn P. Goodrich, Nanospectra Biosciences, Inc., 8285 El Rio Street Suite 150, Houston, TX 77054. Tel: 713-748-3903; Fax: 713-440-9349; E-mail: ggoodrich@nanospectra.com. vasculature cell surfaces. ${ }^{9-14}$ The particle is then exposed to an external or proximate energy source to create a localized hyperthermia to ablate the tumor. The particle serves to transduce the applied energy into heat, and the selectivity of the accumulation of the particle in the tumor serves to focus the transduction at the desired target. It has been demonstrated in animal models that tumor-specific particle accumulation will provide tumor-specific ablation and minimize damage to adjacent tissue or structures. ${ }^{15}$ Particle-based localized hyperthermia has also been demonstrated to enhance the effects of radiation therapy by providing dose-enhancement proximate to the area of particle accumulation. ${ }^{16}$

Near-infrared (NIR) energy is an attractive energy source because human tissue and blood are minimally absorptive in these wavelengths. Particles that absorb near-infrared wavelengths will serve to transduce energy that would otherwise be dissipated. Additionally, commercially available lasers and fiber-optic delivery systems allow the minimally invasive, versatile delivery of energy to most tumor locations. A number of in vitro studies have been published using various nearinfrared absorbing particles for tumor ablation, including nanocages, ${ }^{17,18}$ nanoshells, ${ }^{11,12}$ nanorods, ${ }^{19-25}$ and carbon nanotubes. ${ }^{10}$ The extension of this work into in vivo models

$1083-3668 / 2010 / 15(1) / 018001 / 8 / \$ 25.00$ ○ 2010 SPIE 

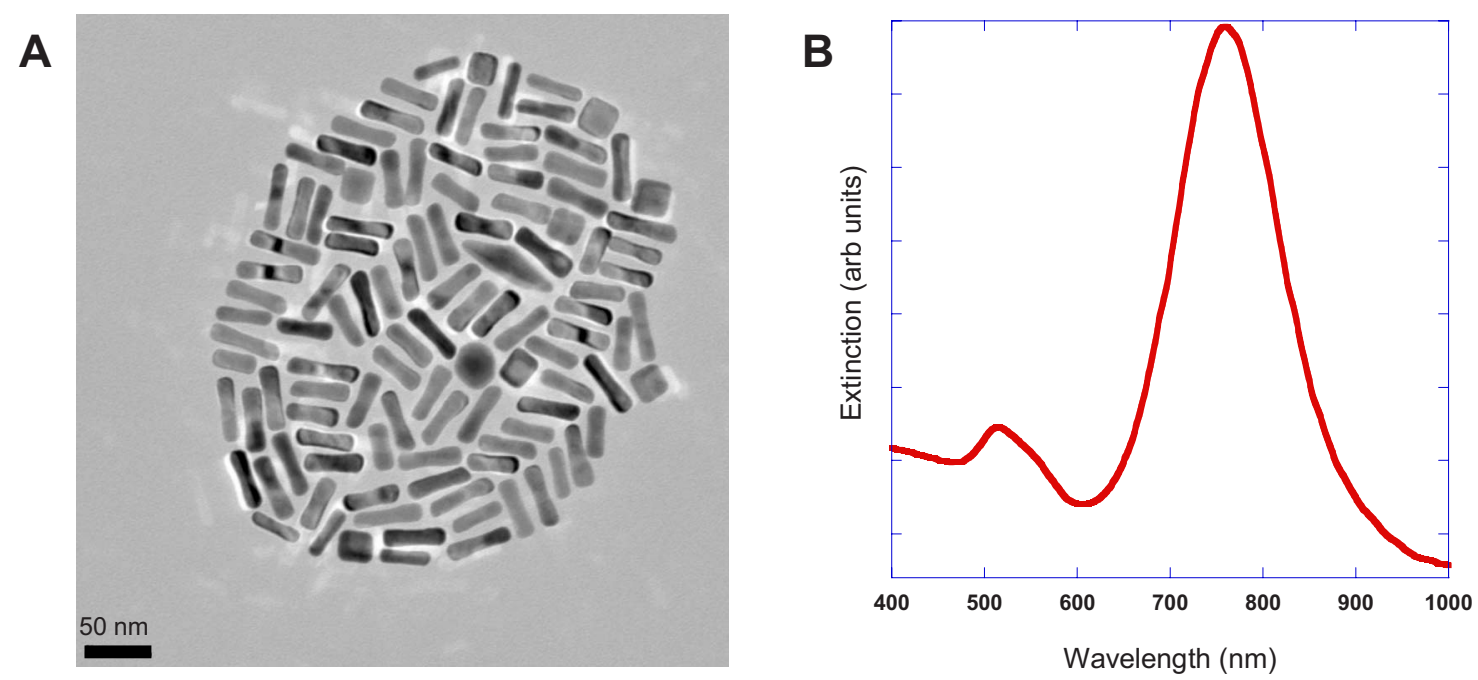

Fig. 1 (a) TEM image of gold nanorods used in this work. Average rod size was determined to be $44.7 \pm 5.4$ nm by $14.3 \pm 1.8$. Non-rod-shaped particles accounted for $\sim 10 \%$ of the particles observed. (b) The optical extinction spectrum of the nanorod particles used is shown in the right panel. All concentrations were standardized to an optical density at $800 \mathrm{~nm}$, the approximate laser wavelength used.

was previously demonstrated by West et al. using gold nanoshells ${ }^{26,27}$ by El-Sayed et al. ${ }^{28}$ and von Maltzhan et al. ${ }^{25}$ using gold nanorods, and by $\mathrm{Lu}$ et al. ${ }^{29}$ using hollow nanoshells.

Recent theoretical evaluations of the thermal efficiency of gold nanorods have generated a significant interest in the use of these particles for photothermal applications because of their relatively small size and high theoretical absorption coefficients. ${ }^{30}$ However, one of the major concerns with gold nanorods has been the reported cytotoxicity of the surfactant cetyltrimethylammonium bromide (CTAB) used in the manufacture of the nanorods, ${ }^{31-33}$ and the nonspecific accumulation that may occur as a result of residual CTAB. ${ }^{34,35}$ While there are previous studies of the biodistribution of gold nanorods ${ }^{36}$ and preliminary efficacy studies, ${ }^{25,28}$ these reports do not evaluate the biodistribution, tumor accumulation, acute toxicity, and efficacy of photothermal ablation in a single study using one manufacturing process. One of the primary differences in the manufacturing process used in this report is the use of diafiltration to remove process residuals. Most published studies used centrifugation as the method for removing excess CTAB from the solution. However, centrifugation is a very costly method for the cleaning of the large volumes of materials that would be needed in a commercial therapy in humans. Diafiltration is easily scalable and can be used to process extremely large volumes of particles. In this work, we report the biodistribution of gold nanorods in healthy animals, the efficacy of photothermal treatment, and the toxicity of the particles at 60 days post-infusion using material manufactured using this process.

\section{Materials and Methods}

\subsection{Synthesis of PEGylated Gold Nanorods}

$\mathrm{HAuCl}_{4} \cdot 3 \mathrm{H}_{2} \mathrm{O}, \mathrm{CTAB}, \mathrm{NaBH}_{4}, \mathrm{AgNO}_{3}$, and ascorbic acid were purchased from Sigma-Aldrich and were used as received with no further modification or purification. Nanorods were synthesized using the method developed by Jana et al. ${ }^{37}$ with minor changes. In brief, gold seed particles were prepared by adding $250 \mu \mathrm{L}$ of $10 \mathrm{mM} \mathrm{HAuCl}_{4} \cdot 3 \mathrm{H}_{2} \mathrm{O}$ to $7.5 \mathrm{~mL}$ of $100 \mathrm{mM}$ CTAB with brief, gentle mixing. $600 \mu \mathrm{L}$ of freshly prepared, ice-cold $10 \mathrm{mM} \mathrm{NaBH} 4$ solution was added, followed by mixing for $2 \mathrm{~min}$. The nanorod growth solution was prepared by adding $40 \mathrm{~mL}$ of $100 \mathrm{mM}$ CTAB, $1.7 \mathrm{~mL}$ of $10 \mathrm{mM} \mathrm{HAuCl} \mathrm{H}_{4} \cdot 3 \mathrm{H}_{2} \mathrm{O}$, and $250 \mu \mathrm{L}$ of $10 \mathrm{mM}$ $\mathrm{AgNO}_{3}$ followed by $270 \mu \mathrm{L}$ of $100 \mathrm{mM}$ ascorbic acid. To initiate nanorod growth, $840 \mu \mathrm{L}$ of the seed solution was added to the growth solution, mixed gently, and left still for $40 \mathrm{~min}$. Excess reactants were removed by centrifugation and resuspension in DI water. The size and distribution of the nanorods was evaluated by transmission electron microscope (TEM) [Fig. 1(a)]. The nanorods were PEGylated by the addition of $1 \mathrm{mM}$ thiol-terminated methoxypoly(ethylene glycol) (mPEG-SH) (Laysan Bio) which was left to stir overnight. The final PEGylated rod solution was cleaned by diafiltration of the solution into DI $\mathrm{H}_{2} \mathrm{O}$. After cleaning, the particles were transferred via diafiltration into a $10 \%(\mathrm{w} / \mathrm{v})$ solution of trehalose to make the solution iso-osmotic with the blood. The final particles showed a strong plasmon resonance centered on $780 \mathrm{~nm}$ [Fig. 1(b)]. Residual CTAB content was estimated using elemental analysis of bromine content in the nanorod solution. Bromine content is an indirect measurement of the residual CTAB content of the solution. Using the measured concentration of bromine in the solution and assuming that each bromine is associated with a molecule of CTAB, it is possible to estimate the molar concentration of CTAB in the solution. Based on this number, we can estimate the solution concentration of CTAB at $3.2 \mu \mathrm{g} / \mathrm{mL}$ in the material used in these studies.

\subsection{Animal Model and Inoculation with Tumor Cells}

57 female Balb/c mice, 5 to 6 weeks of age (Taconic, Hudson, New York) weighing $\sim 16$ to $20 \mathrm{~g}$ were used in this study. For the tumor accumulation study, 9 animals were inoculated subcutaneously on the flank with $50 \mu \mathrm{L}$ 
$\left(8 \times 10^{6}\right.$ cells per $\left.\mathrm{ml}\right)$ of CT26.wt murine colon carcinoma (ATCC) suspended in PBS. For the photothermal efficacy study, 36 mice were inoculated subcutaneously on the flank with $50 \mu \mathrm{L}\left(8 \times 10^{6}\right.$ cell per $\left.\mathrm{ml}\right)$ of CT26.wt murine colon carcinoma. Once the tumor reached a diameter of $\sim 7 \mathrm{~mm}$ in any two directions, the animals were utilized. Of the 9 mice inoculated for the tumor accumulation study, 9 developed usable tumors. Of the 36 innoculated for the thermal efficacy study, 27 developed a treatable tumor. Twelve animals without tumors were used for the biodistribution studies. All animals were housed and all experiments were conducted in accordance with the Guide for Care and Use of Laboratory Animals in Nanospectra's AAALAC-accredited animal facility.

\subsection{Particle Biodistribution}

At 1,7 , and 28 days post-infusion, the animals were sacrificed, and the following tissues were collected for analysis: The brain, heart, lungs, kidneys, liver, spleen, and lymph nodes were taken in their entirety. Samples of the blood and bone were also collected for analysis. The tissue samples were weighed, placed in sample vials, and dessicated. The desiccated tissue, blood samples, or reference materials were analyzed by neutron activation analysis at the University of Texas at Austin (UT-Austin). This method has been shown previously to have sensitivity at 70 picograms of Au per gram of tissue. ${ }^{38}$ Samples and gold standard solutions were placed in the rotary specimen rack of the Mark II TRIGA research reactor for $1 \mathrm{~h}$ at $100 \mathrm{~kW}$. After a nominal one-week decay, the samples were counted in a close geometry using the Compton-suppression system. After counting, the samples were analyzed by UT-Austin for total gold content using a neutron activation analysis package.

\subsection{Rate of Particle Accumulation in Tumor}

Nine tumored mice were given an infusion via the tail vein of gold nanorods $(4.5 \mathrm{ml} / \mathrm{kg}$ of $100 \mathrm{OD}$ material). At each of the 16,24 , and $48 \mathrm{~h}$ post-infusion time points, three mice were sacrificed. Their tumors were harvested, weighed, and dessicated prior to being sent for NAA analysis for gold content.

\subsection{Photothermal Therapy}

Twenty-four hours after a nanorod infusion, the animal was anesthetized with a combination of ketamine/xylazine/ acepromazine. A cooled optical diffusing fiber with an isotropic diffusing tip of $1 \mathrm{~cm}$ (LDF-10, BioTex, Inc., Houston, Texas) was inserted percutaneously under the tumor, and an 808-nm laser (Diomed 15-plus, Diomed, Inc., Cambridge, UK) was used to illuminate the tumor area for $180 \mathrm{~s}$ at $3.5-\mathrm{W}$ average power. A hypodermic needle thermocouple (HYP-130-1/2-T-G-60-SMP-M, Omega Engineering) was placed under the tumor and parallel to the laser fiber to measure the hyperthermia achieved, and the baseline body temperature was recorded using a rectal probe with a digital thermometer. Tumor size was measured three times weekly following therapy. Ellipsoidal tumor volume was calculated using the expression $V=a^{*} b^{*} c^{*}(\pi / 6)$. For all groups, when tumors reached $500 \mathrm{~mm}^{3}$, animals were euthanized. Animals with a complete response were monitored for at least 60 days after treatment.

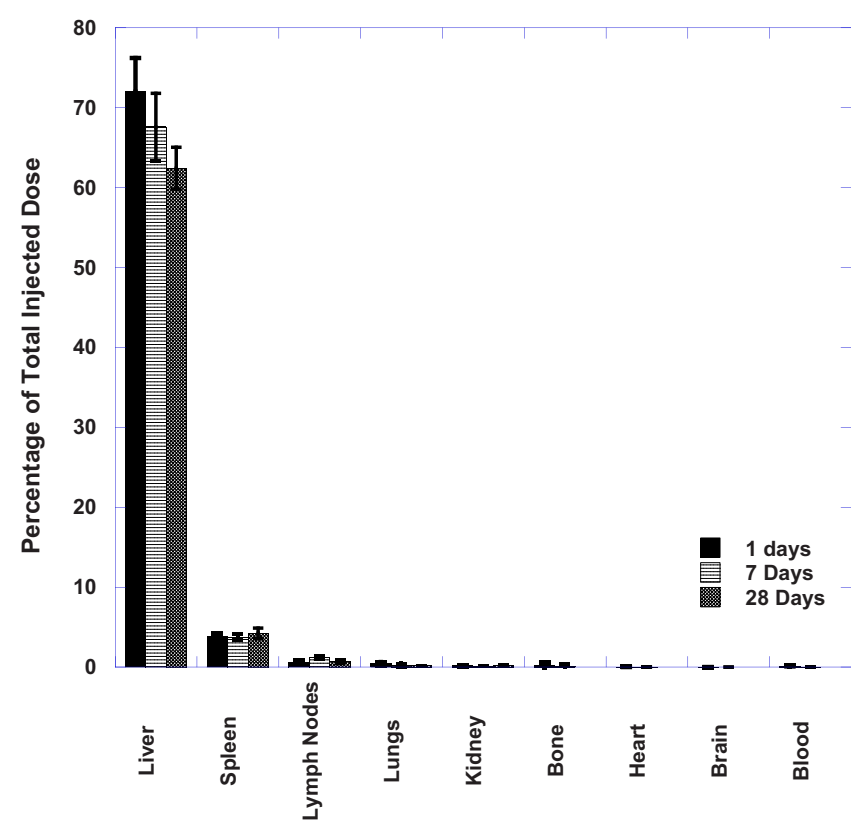

Fig. 2 Gold content plotted as percentage of total infused dose at 1, 7, and 28 days after nanorod infusion as measured by neutron activation analysis. Error bars represent the standard deviation in the measurement.

\section{Results}

\subsection{Biodistribution of Infused Nanorods}

To determine the biodistribution of the gold nanorods after infusion, $12 \mathrm{Balb} / \mathrm{c}$ mice received a tail vein infusion via catherization of PEGylated gold nanorods standardized to $6 \mathrm{~mL} / \mathrm{kg}$ body weight at an optical density of 50 (approximately $6.5 \times 10^{12}$ nanorods per $\mathrm{ml}$ ). Other studies with mice of this strain, comparably sourced and housed, have demonstrated insignificant background gold content. ${ }^{38}$ At 1, 7, and 28 days post-infusion, 4 mice were euthanized, and blood and major organs and selected tissue samples were harvested for neutron activation analysis. Figure 2 shows the percentage of total infused dose ( $\mu \mathrm{g}$ gold per tissue/total $\mu \mathrm{g}$ gold infused) in the major organs at 1, 7, and 28 days post infusion. Figure 2 indicates that the organs of the reticuloendothelial systemthe liver, spleen, and lymph nodes-had substantial nanorod accumulations. There was an insubstantial accumulation of gold in the lungs, kidney, heart, and brain.

\subsection{Nanorod Accumulation in Tumor}

The gold nanorods are expected to accumulate in tumors through fenestrations in the vasculature of a rapidly growing tumor. The rate of particle accumulation will determine the optimal time for treatment post-infusion. To study the rate of particle accumulation in tumors, PEGylated gold nanorods were concentrated to an optical density of 100 and infused into the tail vein of nine tumored Balb/c mice at a dose of $4.5 \mathrm{~mL} / \mathrm{kg}$ body weight (approximately $2 \times 10^{13}$ nanorods per $\mathrm{ml}$ ). Three animals per time point were sacrificed at $16 \mathrm{~h}$, $24 \mathrm{~h}$, and $48 \mathrm{~h}$ after infusion. The tumor was extracted, desiccated, and submitted for neutron activation analysis along with a desiccated sample of the infused nanorod solution as a standard of gold content infused. The gold concentration in 


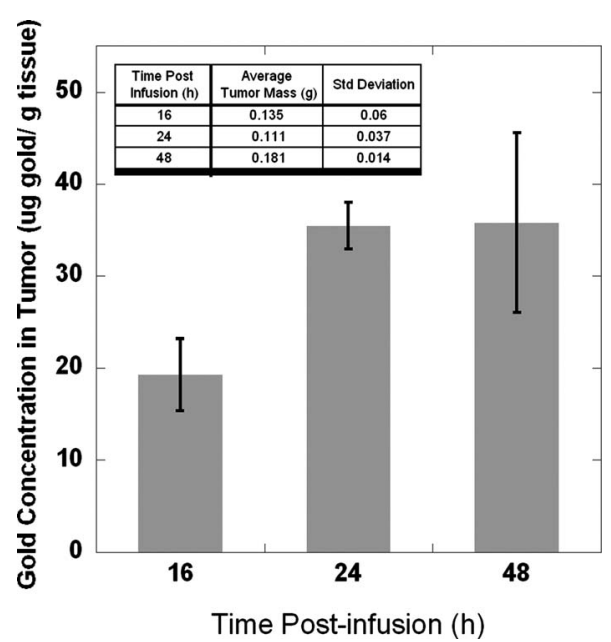

Fig. 3 Gold concentration in tumors in $\mu$ g gold per gram of tissue after infusion with $4.5 \mathrm{~mL} / \mathrm{kg}$ of 100 OD nanorods. Error bars represent the standard deviation in the measurement. Inset table shows the average tumor mass for each sacrifice point with the standard deviation.

the tumor samples ( $\mu \mathrm{g}$ gold per gram of tumor) is shown in Figure 3 as a function of time after infusion. The mean gold concentration increases from $16 \mathrm{~h}$ to $24 \mathrm{~h}$ by a factor of $\sim 1.8 \times$. There is no significant increase in the gold concentration seen from $24 \mathrm{~h}$ to $48 \mathrm{~h}$ post-infusion.

\subsection{Photothermal Therapy Treatment}

Tumored animals were assigned randomly to four groups: nanorod-assisted photothermal therapy, $N=9$; nanorod-only (no laser application) control, $N=7$; laser-only (vehicle infusion, no nanorods) control, $N=6$; and untreated controls, $N$ $=5$. The photothermal therapy group received a tail vein infusion via catherization of PEGylated gold nanorods standardized to $6 \mathrm{~mL} / \mathrm{kg}$ body weight at an optical density of 20 at $800 \mathrm{~nm}$ (approximately $2.6 \times 10^{12}$ nanorods per $\mathrm{ml}$ ). The nanorod-only control group received a comparable tail vein infusion of nanorods as the treatment group, but no laser illumination. The laser-only control group received an infusion of the vehicle (10\% trehalose) but no nanorods, and then a percutaneous laser illumination of the tumor similar to the treatment group. The untreated control group did not receive an infusion or laser application.

The temperature profiles of the nanorod-treated animals versus laser-only controls as measured by the hypodermic thermocouple inserted near the laser fiber are shown in Figure 4. The average measured temperature in both groups increased during the 180-s treatment period. The average maximum temperature difference for nanorod-infused laser treated animals was $\sim 32.1{ }^{\circ} \mathrm{C} \pm 9.0^{\circ} \mathrm{C}$. The average maximum temperature difference for the trehalose infused laser irradiated animals was $\sim 15.3{ }^{\circ} \mathrm{C} \pm 2.8^{\circ} \mathrm{C}$. Based on the average subcutaneous temperature in a mouse of approximately $30{ }^{\circ} \mathrm{C}$, we observe a maximum temperature in tissue of $\sim 62 \pm 9.04{ }^{\circ} \mathrm{C}$ for the nanorod-assisted therapy, and a maximum temperature in tissue in the laser-only control animals was $\sim 45.3^{\circ} \mathrm{C} \pm 2.8^{\circ} \mathrm{C}$.

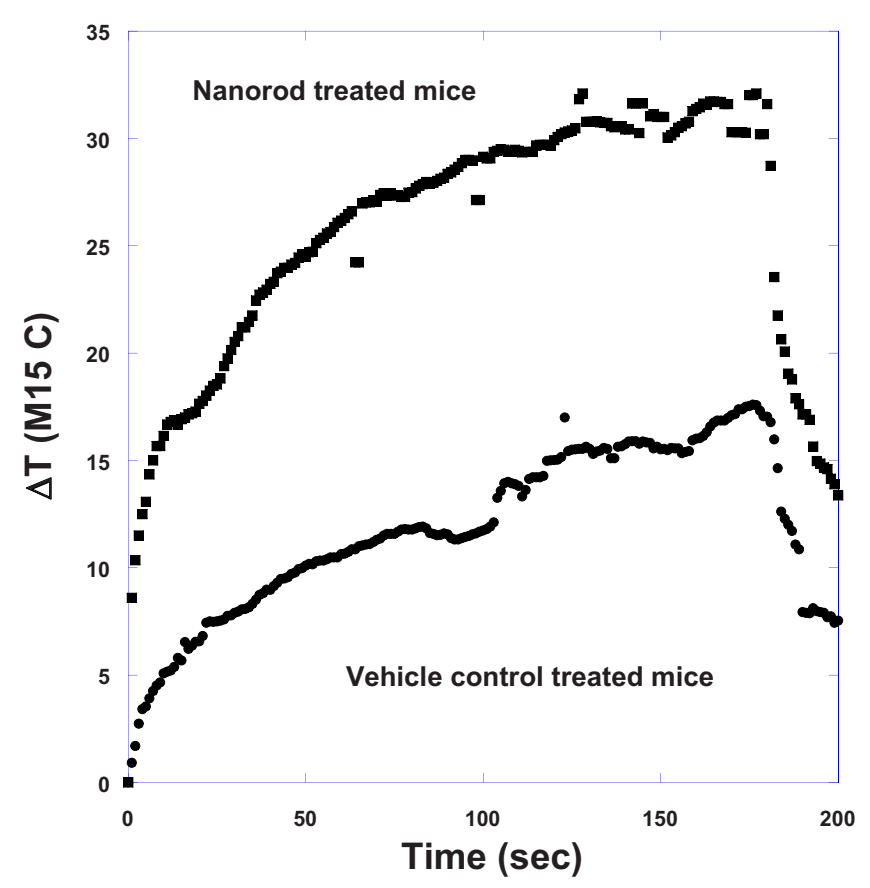

Fig. 4 Thermal measurements in tissue proximal to the laser illumination site. The average value for the animals in each group is shown. The average maximum temperature difference for nanorod-infused laser-treated animals was $\Delta T=32.1{ }^{\circ} \mathrm{C} \pm 9.0^{\circ} \mathrm{C}$. The average maximum temperature difference for the trehalose-infused laser-irradiated animals was $\Delta T=15.3^{\circ} \mathrm{C} \pm 2.8^{\circ} \mathrm{C}$.

The survival curve for all four groups is plotted in Figure 5. The survival time comparison was augmented with a Kaplan-Meier survival analysis to generate confidence intervals for survival times. ${ }^{39}$ The mean survival time for the "no treatment" group was 8 days, with a 95\% confidence interval of 5.1 to 10.9 days. The mean survival time for the "laser illumination only" group was 9.5 days, with a $95 \%$ confidence interval of 7.7 to 11.3 days. The mean survival time for the "nanorods only" group was 9.7 days, with a $95 \%$ confidence interval of 6.3 to 13.1 days. The mean survival time for the photothermal ablation group was 42.1 days, with a $95 \%$ confidence interval of 30.3 to 53.9 days. At day 60, $44 \%$ of the nanorod-treated mice that received a laser treatment survived, with evidence of a complete ablation of the tumor. The mean survival time of photothermally treated animals was statistically higher $(p<0.001)$ than each control group. Survival of each control group was not statistically different from any other control group.

\subsection{Histopathology of Nanorod-Infused Animals}

Four animals showing complete tumor regression after nanorod infusion followed by laser illumination were sacrificed between 62 and 79 days after infusion, and the major organs were collected for histopathology. Four animals that received nanorods but no laser treatment were sacrificed when their tumor volume exceeded limits (ranging from 8 to 18 days after infusion), and the major organs were collected for histopathology. The major organs of three of the untreated controls were also submitted for histopathology for comparison. The tissues were blocked in paraffin, cut and stained using hemo- 


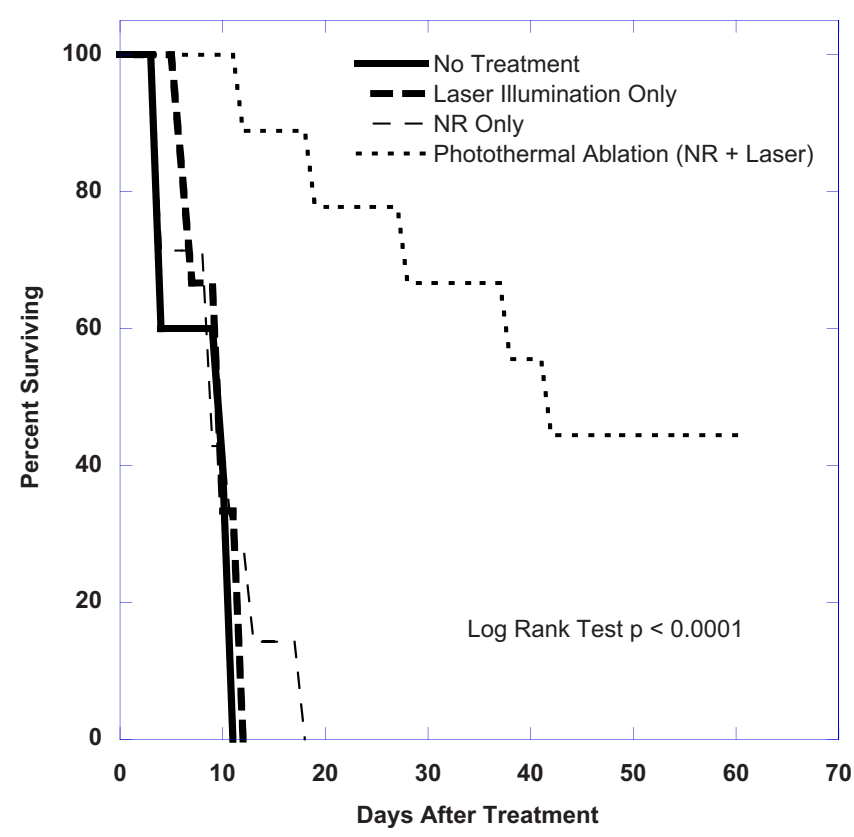

Fig. 5 Survival of tumor-bearing animals by treatment group. Survival of the treatment group was statistically significant from the control groups. The mean survival time for the "no treatment" group was 8 days with a $95 \%$ confidence interval of 5.1-10.9 days. The mean survival time for the "laser illumination only" group was 9.5 days with a $95 \%$ confidence interval of 7.7-11.3 days. The mean survival time for the "nanorods only" group was 9.7 days with a $95 \%$ confidence interval of 6.3-13.1 days. The mean survival time for the photothermal ablation group was 42.1 days with a 95\% confidence interval of $30.3-53.9$ days.

toxylin and eosin (H\&E). The stained slides were sent to be read by Dr. Charles Montgomery, DVM, DACVP, DACLAM (ComPath, Jay, Oklahoma). The following organs were examined histologically: brain, heart, lung, spleen, kidney, adrenal gland, mesenteric lymph nodes, and liver.

In all nanorod-infused animals, a "foreign body," black in color and visible as single dots or aggregates of 5 to 50 dots, was observed in certain tissues. An example of the foreign body is shown in Figure 6. In this image, the foreign body is visible in an $H \& E$ stained section of liver from an animal that

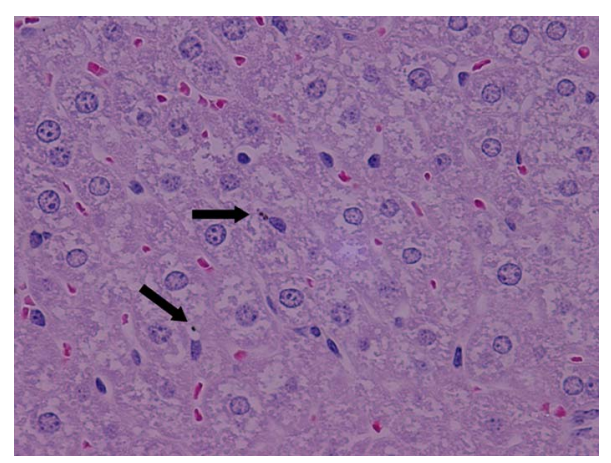

Fig. $6 \mathrm{H} \& \mathrm{E}$ stained liver section from an animal that received an i.v. infusion of Au nanorods. Arrows indicate the presence of the "foreign body" described in the pathology report. As individual nanorods are too small to be observed with optical microscopy, the foreign body is believed to be cluster of nanorods. received a nanorod infusion. Individual nanorods are too small to be seen under optical microscopy; therefore, the foreign bodies observed microscopically are believed to be accumulations or complexes of particles. The incidence, distribution, and grade of severity for inflammatory, degenerative, and proliferative lesions, as well as the presence of foreign bodies in tissues are shown in Table 1.

This foreign body was observed in the spleen (7 of 8) and liver (8 of 8 ) of the animals infused with nanorods. These foreign bodies were not observed in the brain, heart, lung, kidney, adrenal gland, or mesenteric lymph nodes. Splenic foreign bodies were located in the perifollicular zone of the lymphoid follicles and were minimal in severity. Liver foreign bodies were observed free in the sinusoids and occasionally in Kupffer cells lining the sinusoids. Chronic inflammatory lesions were observed in the liver associated with the presence of these foreign bodies, and these lesions consisted of infiltrates of lymphocytes, macrophages, and occasional plasma cells. These foci were random in distribution and minimal in severity, and were present in all of the nanorod-infused animals. This type of host response was not present in vehicle control animals.

\section{Discussion}

The development of a clinically useful photothermal ablation technology must evaluate multiple questions. The particle must accumulate with sufficient selectivity in the tumor to confine thermal damage to the tumor, with minimal damage to surrounding healthy tissue. The accumulation rate of the particle in the tumor and the particle's thermal transduction efficiency must be sufficient to allow transduction of an ablative level of energy. This applied energy must have minimal effect on tissue unless the transducing particles are present and must allow focal application to avoid damage to clearance organs such as the liver. Last, the infused particle dose must have a safety profile appropriate for the intended use (e.g., cancer in adults). The results of this study provide a preliminary answer to several of these questions.

It is important to note that broad generalizations regarding nanomaterials are problematic. For example, mean size, size distribution, shape, residual contaminants, and exponents may affect the safety or efficacy of any particular nanomaterial. Additionally, measurements of biodistribution should be evaluated in the context of the precision and direct/indirect nature of the measurement tool utilized. This study utilized gold nanorods approximately $14 \times 45 \mathrm{~nm}$ in size, and smaller or larger nanorods may have different tumor selectivity.

Particle accumulation in the tumor is perhaps the most important deciding factor in designing photothermal therapies. If the particle does not accumulate sufficiently using a particular injected dose to achieve ablative temperatures, the therapy can never be successful. Two important factors involved in the accumulation in tumor are the total particle dose and the rate of particle accumulation in the tumor. In order to determine the level of particle accumulation in this tumor model, accumulation studies were carried out using nanorod solutions concentrated to an optical density of 100 (approximately $2 \times 10^{13}$ nanorods per $\mathrm{ml}$ ). This concentration was chosen because it is comparable to the optical density of previous studies using gold nanorods. ${ }^{28}$ Tumors were harvested 
Goodrich et al.: Photothermal therapy in a murine colon cancer model using near-infrared absorbing gold nanorods

Table 1 Incidence, grade of severity and distribution of lesions in tissues.

\begin{tabular}{|c|c|c|c|c|c|c|}
\hline \multirow[b]{2}{*}{ Organ/lesion } & \multicolumn{2}{|c|}{ Vehicle control } & \multicolumn{2}{|c|}{ Photothermal therapy } & \multicolumn{2}{|c|}{ Nanorod control } \\
\hline & Incidence & $\begin{array}{l}\text { Average } \\
\text { severity }\end{array}$ & Incidence & $\begin{array}{l}\text { Average } \\
\text { severity }\end{array}$ & Incidence & $\begin{array}{l}\text { Average } \\
\text { severity }\end{array}$ \\
\hline Brain & 0 & 0 & 0 & 0 & 0 & 0 \\
\hline $\begin{array}{l}\text { Heart } \\
\text { Mineralization }\end{array}$ & 0 & 0 & $2 / 4$ & 2.0 & $1 / 4$ & 1.0 \\
\hline $\begin{array}{l}\text { Lung } \\
\text { Chronic inflammation }\end{array}$ & 0 & 0 & $1 / 4$ & 1.0 & $1 / 4$ & 2.0 \\
\hline $\begin{array}{l}\text { Spleen } \\
\text { Acid hematin pigment }\end{array}$ & $2 / 3$ & 1.0 & $4 / 4$ & 2.3 & $4 / 4$ & 1.0 \\
\hline $\mathrm{FB}$ & 0 & 0 & $4 / 4$ & 1.0 & $3 / 4$ & 1.0 \\
\hline $\begin{array}{l}\text { Kidney } \\
\quad \text { Mineralization }\end{array}$ & 0 & 0 & 0 & 0 & $1 / 4$ & 1.0 \\
\hline $\begin{array}{l}\text { Adrenal gland } \\
\text { Subcapsular hyperplasia }\end{array}$ & $3 / 3$ & 2.0 & $4 / 4$ & 1.0 & $1 / 4$ & 1.0 \\
\hline Mesenteric lymph node & 0 & 0 & 0 & 0 & 0 & 0 \\
\hline $\begin{array}{l}\text { Liver } \\
\text { Mononuclear cell infiltrate }\end{array}$ & $2 / 3$ & 1.0 & $4 / 4$ & 1.0 & $2 / 4$ & 1.0 \\
\hline $\begin{array}{l}\text { Chronic inflammation, } \\
\text { etiology FB }\end{array}$ & 0 & 0 & $4 / 4$ & 1.0 & $4 / 4$ & 1.0 \\
\hline FB, sinusoids & 0 & 0 & $4 / 4$ & 1.0 & $4 / 4$ & 1.0 \\
\hline
\end{tabular}

$\mathrm{FB}=$ foreign body. Grade of severity: $0=$ no lesion, $1=$ minimal, $2=$ mild, $3=$ moderate, $4=$ marked.

for gold analysis at $16 \mathrm{~h}, 24 \mathrm{~h}$, and $48 \mathrm{~h}$ after infusion. The gold accumulation was determined using NAA. NAA allows for the quantitative determination of gold in the evaluated tissue by comparison to a recognized standard, and has demonstrated sensitivity to 70 picograms per gram. ${ }^{38}$ The results shown in Fig. 3 indicate that significant accumulation was achieved by $24 \mathrm{~h}$ after infusion. This result is consistent with particle accumulations reported by El Sayed et al. ${ }^{28}$ As there was no significant increase in gold content measured after $24 \mathrm{~h}$, this time point was chosen for the photothermal therapy.

In any therapeutic treatment, it is desirable to use the least amount of exogenous material to achieve effective treatment. This is important both to keep the cost of a potential treatment low and to minimize any potential side effects due to the material in the body. A recent study of the thermal transduction efficiency of nanorods and other nanoparticles reports that the thermal transduction efficiency of the gold nanorods is approximately twice that of gold nanoshells for a given optical density. ${ }^{40}$ Based on measurements of gold accumulations from previous nanoshell studies ${ }^{15}$ and the estimated number of gold nanorods determined from the tumor accumulation levels using 100 optical density material, a therapeutically effective particle dose level, i.e., the minimum particle dose that would provide tumor accumulations adequate to achieve ablation, was estimated to be $6 \mathrm{~mL} / \mathrm{kg}$ of particles concentrated to an optical density of 20 . This dose level is significantly lower than that reported by El Sayed et al. for their i.v. experiments (approx $5 \mathrm{ml} / \mathrm{kg}$ at $120 \mathrm{OD}){ }^{28}$

The tumor accumulation studies indicate that there is a significant accumulation of particles in the tumor after a systemic infusion of gold nanorods. To successfully localize the thermal therapy to the tumor, it is also necessary to show selective accumulation in tumors. Our prior experience indicates that the presence of a tumor with a fenestrated vasculature does not materially alter biodistribution of infused particles among other organs because less than $10 \%$ of the total injected dose accumulates in the tumor. Accordingly, the biodistribution of infused nanorods was evaluated in nontumored animals. In addition, the use of nontumored animals would allow the biodistribution study to be extended to 28 days post-infusion to get preliminary information about particle clearance from the body. The gold accumulation in various tissues was determined using NAA analysis and allows the harvest and measurement of whole organs but does not provide for intra-organ information. The results of this study indicate clearance and accumulation at 1, 7, and 28 days after infusion by organs of the reticuloendothelial system, consistent with previous studies of other particles. ${ }^{36,38}$ The largest accumulation of nanoparticles was observed in the liver and spleen, which together account for approximately $75 \%$ of the total injected nanoparticles at 1 day post-injection. There was also a small accumulation of particles $(<1 \%$ total injected dose) seen in the kidney and lymph nodes. None of the other organs or tissues measured, including the heart, lungs, muscle, 
and bone show any significant gold content. The biodistribution indicates a gradual clearance of gold from the liver $(72 \%$ of total dose at 1 day to $62 \%$ of total dose at 28 days). While the gold content decreases in the liver over the 28-day study, the gold content in the other measured organs remains fairly constant. These biodistribution and tumor accumulation results indicate that nanorods of the present size will not extravasate into healthy tissue and must exit the blood stream through tumor vasculature fenestrations in order to be accumulated. As recently demonstrated, the use of different particle geometry (e.g., size, shape) tumor-specific targeting molecules may alter these kinetics, both within the tumor and in nontargeted organs. ${ }^{29}$

The success of a particle-assisted photothermal therapy depends on the particle accumulation and the appropriate laser dosimetry. Too high a laser exposure will cause excessive heating and damage to healthy tissue surrounding the tumor. If the laser dose is too low, then complete ablation may not be achieved, and the tumor may regrow. For this experiment, a laser exposure of 3.5-W average power for $180 \mathrm{~s}$ was chosen, based on our previous studies using gold nanoshells. ${ }^{15,27}$ In order to gauge the appropriateness of the chosen laser exposure using this particle and tumor model, the thermal response to laser treatment was measured for the two test groups subjected to thermal therapy via the use of a thermocouple inserted near the tumor site. The laser dosimetry of $3.5-\mathrm{W}$ average power produced temperature rises sufficient to ablate the tumor proximate to the fiber placement. Observation of the tumors in the nanorod-treated mice that showed incomplete regression indicated that the tumor regrowth was from a location farthest from the placement of the optical fiber. As the thermal data illustrates that ablative temperatures were achieved in the nanorod-treated mice, this would indicate that the placement of the laser fiber may have affected the ablation of the tumors. In this study, we used a substantially lower particle dose than in the study by El-Sayed et al. ${ }^{28}$ While this combination of nanorod dose and laser exposure level was sufficient for ablative effects in all treated animals and a complete ablation in $>40 \%$ of the treated animals, further investigation of dose accumulations are warranted, particularly if safety thresholds will tolerate higher doses of particles.

This study also indicates encouraging results regarding the safety of a residual level of CTAB. CTAB been previously shown to be highly cytotoxic in solution ${ }^{31,41}$ and unPEGylated CTAB-coated nanorods have also evidenced cytotoxic effects in cell culture. ${ }^{32}$ The use of PEG to displace the CTAB from the rod surface has been shown to reduce the observed toxicity of the particles in cell culture assays. ${ }^{32,36,43}$ However, cell culture toxicity assays generally focus on cell death at a 24- to 72-h time points, which may be insufficient time for any residual CTAB to elute off the particle. The present biodistribution study indicates the presence of gold nanorods in the liver and spleen past 28 days. The elemental analysis results indicate that nanorod-infused animals received approximately $0.32 \mu \mathrm{g}$ of CTAB per gram of body weight. The only significant lesion noted by the veterinary pathologist was a minimal incidence of chronic inflammation in the liver. We have observed in studies with gold nanoshells that do not contain CTAB a similar level of chronic inflammation in the liver, so we believe that this inflammation is not due to the levels of residual CTAB. While this inflammation appears to be minor, the presence of particles in the liver at 60 days after infusion would suggest that a longer term chronic toxicity study would be warranted to determine any long-term effects of exposure to the nanorods. Histopathology did not indicate any significant lesions associated with the nanorod or residual CTAB levels.

This study demonstrates the safety and efficacy of the chosen gold nanorod for the photothermal ablation of solid tumors. The infused particles selectively accumulated in the tumor in quantities sufficient to provide significant ablation of the target lesions. There were no signs of acute toxicity from the infused particles through 60 days after infusion. The results suggest that optimization of the particle dose and laser dosimetry may lead to a highly effective therapy. Further study of the long-term retention, clearance, and safety of the particles is required.

\section{Acknowledgments}

The authors would like to acknowledge the contribution of Dr. Jon Schwartz for his laser dosimetry work. The authors would also like to thank Dr. Charles Montgomery, DVM, DACVP, DACLAM of ComPath in Jay, Oklahoma, for performing the histopathology analysis. All authors were employees and equity holders of Nanospectra Biosciences during the performance of this study, and Nanospectra may benefit financially from commercialization of this work.

\section{References}

1. A. Carpentier, R. J. McNichols, R. J. Stafford, J. Itzcovitz, J. P. Guichard, D. Reizine, S. Delaloge, E. Vicaut, D. Payen, A. Gowda, and B. George, "Real-time magnetic resonance-guided laser thermal therapy for focal metastatic brain tumors," Neurosurgery 63(1), 21-28 (2008).

2. M. Kangasniemi, R. J. McNichols, J. A. Bankson, A. Gowda, R. E. Price, and J. D. Hazle, "Thermal therapy of canine cerebral tumors using a 980-nm diode laser with MR temperature-sensitive imaging feedback," Lasers Surg. Med. 35(1), 41-50 (2004).

3. R. Puls, S. Langner, C. Rosenberg, K. Hegenscheid, J. P. Kuehn, K. Noeckler, and N. Hosten, "Laser ablation of liver metastases from colorectal cancer with MR thermometry: 5-year survival," J. Vasc. Interv. Radiol. 20(2), 225-234 (2009).

4. G. S. Gazelle, S. N. Goldberg, L. Solbiati, and T. Livraghi, "Tumor ablation with radio-frequency energy," Radiology 217(3), 633-646 (2000).

5. A. N. Mirza, B. D. Fornage, N. Sneige, H. M. Kuerer, L. A. Newman, F. C. Ames, and S. E. Singletary, "Radio-frequency ablation of solid tumors," Cancer J. 7(2), 95-102 (2001).

6. O. Ishida, K. Maruyama, K. Sasaki, and M. Iwatsuru, "Sizedependent extravasation and interstitial localization of polyethyleneglycol liposomes in solid tumor-bearing mice," Int. J. Pharm. 190(1), 49-56 (1999).

7. H. Maeda, "The enhanced permeability and retention (EPR) effect in tumor vasculature: the key role of tumor-selective macromolecular drug targeting," Adv. Enzyme Regul. 41, 189-207 (2001).

8. H. Maeda, J. Fang, T. Inutsuka, and Y. Kitamoto, "Vascular permeability enhancement in solid tumor: various factors, mechanisms involved, and its implications," Int. J. Immunopharmacol 3(3), 319$328(2003)$

9. N. J. Durr, T. Larson, D. K. Smith, B. A. Korgel, K. Sokolov, and A. Ben-Yakar, "Two-photon luminescence imaging of cancer cells using molecularly targeted gold nanorods," Nano Lett. 7(4), 941-945 (2007).

10. N. W. S. Kam, M. O'Connell, J. A. Wisdom, and H. Dai, "Carbon nanotubes as multifunctional biological transporters and near-infrared agents for selective cancer cell destruction," Proc. Natl. Acad. Sci. U.S.A. 102, 11600-11605 (2005).

11. C. Loo, A. R. Lowery, N. J. Halas, J. L. West, and R. Drezek, "Im- 
munotargeted nanoshells for integrated cancer imaging and therapy," Nano Lett. 5(4), 709-711 (2005).

12. A. R. Lowery, A. M. Gobin, E. S. Day, N. J. Halas, and J. L. West, "Immunonanoshells for targeted photothermal ablation of tumor cells," Int. J. Nanomed. 1(2), 149-154 (2006).

13. R. S. Norman, J. W. Stone, A. Gole, C. J. Murphy, and T. L. SaboAttwood, "Targeted photothermal lysis of the pathogenic bacteria, Pseudomonas aeruginosa, with gold nanorods," Nano Lett. 8(1), 302-306 (2008).

14. A. K. Oyelere, P. C. Chen, X. H. Huang, I. H. El-Sayed, and M. A. El-Sayed, "Peptide-conjugated gold nanorods for nuclear targeting," Bioconjugate Chem. 18, 1490-1497 (2007).

15. J. A. Schwartz, A. M. Shetty, R. E. Price, R. J. Stafford, J. C. Wang, R. K. Uthamanthil, K. Pham, R. J. McNichols, C. L. Coleman, and J. D. Payne, "Feasibility study of particle-assisted laser ablation of brain tumors in orthotopic canine model," Cancer Res. 64(4), 16591667 (2009).

16. P. Diagaradjane, A. Shetty, J. C. Wang, A. M. Elliott, J. Schwartz, S. Shentu, H. C. Park, A. Deorukhkar, R. J. Stafford, S. H. Cho, J. W. Tunnell, J. D. Hazle, and S. Krishnan, "Modulation of in vivo tumor radiation response via gold nanoshell-mediated vascular-focused hyperthermia: characterizing an integrated antihypoxic and localized vascular disrupting targeting strategy," Nano Lett. 8(5), 1492-1500 (2008).

17. J. Y. Chen, D. L. Wang, J. F. Xi, L. Au, A. Siekkinen, A. Warsen, Z. Y. Li, H. Zhang, Y. N. Xia, and X. D. Li, "Immuno gold nanocages with tailored optical properties for targeted photothermal destruction of cancer cells," Nano Lett. 7, 1318-1322 (2007).

18. J. Y. Chen, B. Wiley, Z. Y. Li, D. Campbell, F. Saeki, H. Cang, L. Au, J. Lee, X. D. Li, and Y. N. Xia, "Gold nanocages: engineering their structure for biomedical applications," Adv. Mater. 17(18), 22552261 (2005).

19. T. S. Hauck, T. L. Jennings, T. Yatsenko, J. C. Kumaradas, and W. C. W. Chan, "Enhancing the toxicity of cancer chemotherapeutics with gold nanorod hyperthermia," Adv. Mater. 20(20), 3832-3838 (2008).

20. X. H. Huang, I. H. El-Sayed, W. Qian, and M. A. El-Sayed, "Cancer cell imaging and photothermal therapy in the near-infrared region by using gold nanorods," J. Am. Chem. Soc. 128(6), 2115-2120 (2006).

21. Y. F. Huang, K. Sefah, S. Bamrungsap, H. T. Chang, and W. Tan, "Selective photothermal therapy for mixed cancer cells using aptamer-conjugated nanorods," Langmuir 24(20), 11860-11865 (2008).

22. T. B. Huff, L. Tong, Y. Zhao, M. N. Hansen, J. X. Cheng, and A. Wei, "Hyperthermic effects of gold nanorods on tumor cells," Nanomedicine 2(1), 125-132 (2007).

23. H. Takahashi, T. Niidome, A. Nariai, Y. Niidome, and S. Yamada, "Gold nanorod-sensitized cell death: microscopic observation of single living cells irradiated by pulsed near-infrared laser light in the presence of gold nanorods," Chem. Lett. 35(5), 500-501 (2006).

24. L. Tong, Y. Zhao, T. B. Huff, M. N. Hansen, A. Wei, and J. X. Cheng, "Gold nanorods mediate tumor cell death by compromising membrane integrity," Adv. Mater. 19(20), 3136-3141 (2007).

25. G. von Maltzahn, J.-H. Park, A. Agrawal, N. K. Bandaru, S. K. Das, M. J. Sailor, and S. N. Bhatia, "Computationally guided photothermal tumor therapy using long-circulating gold nanorod antennas," Cancer Res. 69(9), 3892-3900 (2009).

26. L. R. Hirsch, R. J. Stafford, J. A. Bankson, S. R. Sershen, B. Rivera, R. E. Price, J. D. Hazle, N. J. Halas, and J. L. West, "Nanoshell- mediated near-infrared thermal therapy of tumors under magnetic resonance guidance," Proc. Natl. Acad. Sci. U.S.A. 100(23), 1354913554 (2003).

27. D. P. O'Neal, L. R. Hirsch, N. J. Halas, J. D. Payne, and J. L. West, "Photo-thermal tumor ablation in mice using near infrared-absorbing nanoparticles," Cancer Lett. 209(2), 171-176 (2004).

28. E. B. Dickerson, E. C. Dreaden, X. H. Huang, I. H. El-Sayed, H. H. Chu, S. Pushpanketh, J. F. McDonald, and M. A. El-Sayed, "Gold nanorod assisted near-infrared plasmonic photothermal therapy (PPTT) of squamous cell carcinoma in mice," Cancer Lett. 269(1), 57-66 (2008).

29. W. Lu, C. Y. Xiong, G. D. Zhang, Q. Huang, R. Zhang, J. Z. Zhang, and C. Li, "Targeted photothermal ablation of murine melanomas with melanocyte-stimulating hormone analog-conjugated hollow gold nanospheres," Clin. Cancer Res. 15(3), 876-886 (2009).

30. P. K. Jain, K. S. Lee, I. H. El-Sayed, and M. A. El-Sayed, "Calculated absorption and scattering properties of gold nanoparticles of different size, shape, and composition: applications in biological imaging and biomedicine," J. Phys. Chem. B 110(14), 7238-7248 (2006).

31. R. Cortesi, E. Esposito, E. Menegatti, R. Gambari, and C. Nastruzzi, "Effect of cationic liposome composition on in vitro cytotoxicity and protective effect on carried DNA," Int. J. Pharm. 139(1-2), 69-78 (1996).

32. T. S. Hauck, A. A. Ghazani, and W. C. W. Chan, "Assessing the effect of surface chemistry on gold nanorod uptake, toxicity, and gene expression in mammalian cells," Small 4(1), 153-159 (2008).

33. H. Takahashi, Y. Niidome, T. Niidome, K. Kaneko, H. Kawasaki, and S. Yamada, "Modification of gold nanorods using phosphatidylcholine to reduce cytotoxicity," Langmuir 22(1), 2-5 (2006).

34. E. E. Connor, J. Mwamuka, A. Gole, C. J. Murphy, and M. D. Wyatt, "Gold nanoparticles are taken up by human cells but do not cause acute cytotoxicity," Small 1(3), 325-327 (2005).

35. T. B. Huff, M. N. Hansen, Y. Zhao, J. X. Cheng, and A. Wei, "Controlling the cellular uptake of gold nanorods," Langmuir 23(4), 15961599 (2007).

36. T. Niidome, M. Yamagata, Y. Okamoto, Y. Akiyama, H. Takahashi, T. Kawano, Y. Katayama, and Y. Niidome, "PEG-modified gold nanorods with a stealth character for in vivo applications," J. Controlled Release 114(3), 343-347 (2006).

37. N. R. Jana, L. Gearheart, and C. J. Murphy, "Wet chemical synthesis of high aspect ratio cylindrical gold nanorods," J. Phys. Chem. B 105(19), 4065-4067 (2001).

38. W. D. James, L. R. Hirsch, J. L. West, P. D. O'Neal, and J. D. Payne, "Application of INAA to the build-up and clearance of gold nanoshells in clinical studies in mice," J. Radioanal. Nucl. Chem. 271(2), 455-459 (2007).

39. E. L. Kaplan and P. Meier, "Nonparametric estimation from incomplete observations," J. Am. Stat. Assoc. 53, 457-481 (1958).

40. J. R. Cole, N. A. Mirin, M. W. Knight, G. P. Goodrich, and N. J. Halas, "Photothermal efficiencies of nanoshells and nanorods for clinical therapeutic applications," J. Phys. Chem. C 113(28), 1209012094 (2009).

41. C. L. Bean, S. M. Galloway, and M. O. Bradley, "Evaluation of three in vitro assays for assessment of membrane damage by surfactants," In Vitro Toxicol. 4(2), 133-144 (1991).

43. H. Liao and J. H. Hafner, "Gold nanorod bioconjugates," Chem. Mater. 17, 4636-4641 (2005). 